\title{
NOTE ON THE GRADUATION OF GERBER BUTYROMETERS.
}

\author{
By H. DROOP RICHMOND, F.I.C.
}

(Read at the Meeting, November 6, 1918.)

In the formula given by Day and Grimes (Analyst, 1918, 123) the expression 1-000027 $(T-15)$ is redundant, as it reduces the volume from that at $T^{\circ}$ to $15^{\circ}$, and this is already done by using $D_{T}=$ apparent density of fat in glass at $T^{\circ}$; the expression "apparent density in glass" means that the true density is already corrected for the expansion of the glass, and it is unnecessary to make this correction again, and consequently Day and Grimes' figures are all slightly too low.

They state that measurements of the weight of milk at $11^{\circ} \mathrm{C}$. and $21^{\circ} \mathrm{C}$. were practically identical, which is quite in accord with my own observations, but they are in error in stating that this effect was less than required by theory, as they have apparently overlooked the fact that the decrease in density with temperature is nearly counterbalanced by the decrease in surface energy.

Day and Grimes state that the value for "expansion" given by me is 0.000863 , but it is calculated on the specific volume instead of unity; this is quite correct, and expansion should be calculated as a volume expression, and when expressed as spectic volume (i.e., volume of $1 \mathrm{grm}$.) it is quite independent of the value of specific volume at any temperature; if calculated to the volume taken as unity, it should obviously be taken as a ratio to the volume at the temperature taken as the startingpoint. Their calculation that my coefficient of expansion (in terms of specific volume) is 0.000745 between $38.5^{\circ}$ and $100^{\circ} \mathrm{C}$. is incorrect, and their so-called coefficients of expansion are really the decreases of the weights of 1 c.c. taking the weight of $1 \mathrm{c} \mathrm{c}$. at $30^{\circ} \mathrm{C}$. as unity, rather a meaningless expression, and certainly not a " coefficient of expansion."

The use of this expression has led them to miscalculate figures which they give as "Calculated from Richmond's data," as the following table will show:

\begin{tabular}{|c|c|c|c|c|c|}
\hline \multirow{4}{*}{$\begin{array}{l}\text { Milk fat } \\
\text { Calculated by } \\
\begin{array}{l}\text { Grimes } \\
\text { Calculated correctly }\end{array} \\
\text { Cand }\end{array}$} & \multicolumn{3}{|c|}{ True Specific Gravity } & \multicolumn{2}{|c|}{$\begin{array}{l}\text { Apparent Specific Gravity in } \\
\text { Glass compared with Water } \\
\text { at } 155^{\circ} \mathrm{C} \text {. }\end{array}$} \\
\hline & $30^{\circ} \mathrm{C}$ & $65^{\circ} \mathrm{C}$ & $100^{\circ} \mathrm{C}$. & $55^{\circ} \mathrm{C}$ & $65^{\circ} \mathrm{C}$. \\
\hline & 0.9109 & 0.8872 & $0 \cdot 8637$ & 0.8966 & 0.8899 \\
\hline & 0.9113 & $0 \cdot 8868$ & 08637 & $0 \cdot 8956$ & 0.8888 \\
\hline
\end{tabular}

I note that they find the volume of the menisous in round scale butyrometers to be 0.05 division, while $I$ had assumed it to be 0.08 division from measurements ; I accept their measurement as correct, and this reduces my ratio of the volume of Gerber fat to true fat from 1.025 to 1.015 . 
With reference to the temperature of reading, I feel confident that in my experiments the temperature of the fat when read was approximately $70^{\circ} \mathrm{C}$., as not only was a steam turbine used which kept the bottles hot till read, but they were read in close proximity to a lamp, and in some cases in a jacketed tube (devised to measure the expansion of fat in the neck). Despite the fact that they found a considerable change in the fat on analysis, they make the assumption that the volume changes are small, and compensate one another closely.

Their analysis of the Gerber fat, though confirming mine in most particulars, differs in that I found a lower density for Gerber fat than milk fat, while they find a higher; their quotation from Lewkowitsch as to increased molecular volume would rather lead one to expect a lower density, as small quantities of amyl alcohol and especially amyl esters are present. I would mention that my deduction that the weight of the fat increases about 3 per cent. is borne out entirely by the observations made by Rosier and myself (ANALYST, 1899, 24, 172), in which we found that the fat extracted by petroleum ether was about to this extent greater in weight than the truth, and was reduced to the true weight by washing with water.

But, after all, the calculations that both Day and Grimes and myself have made are only incidental and confirmatory, and it is really on the agreement between Gerber results and gravimetric analyses that our respective conclusions are based.

On this point we differ slightly, as while I find that bottles graduated 1 division $=0.126$ c.c. gives the most accurate results (and the few results I quoted could be multiplied a hundred-fold) they find that $0 \cdot 124$ c.c. agrees with their results. This is perhaps due to a slight difference of conditions, I working at rather a higher temperature than they, perhaps whirling longer (usually ten minutes, at least 1,000 rev. per minute in a steam turbine), and using possibly a stronger acid, as I always employ acid of sp. gr. 1.825 (the upper limit).

There is also another possible cause, a difference in our method of carrying out the gravimetric analyses; I used the procedure described on pp. 110, 111 of " Dairy Chemistry, 1914," for the Adams, and on p. 119 for the Gottlieb method, and it is possible that Messrs. Day and Grimes omitted the tare in the Adams, and the petroleum ether treatment in the Gottlieb, which would cause their results to be slightly high, and account for a considerable portion of our difference.

Incidentally I would confirm the observation that the results by the RoseGottlieb method using an aliquot may be low; the reason is of course due to the fact that the solvent is soluble to some extent in the aqueous alcoholic layer, and this causes a slight solubility of the fat.

Finally, I would point out one conclusion that they have missed, and which, though I did not state it, follows from the results I gave, and that is that the decreased weight of milk delivered by an 11 c.c. pipette as the fat increases partly compensates for the volume occupied by the meniscus; the decrease of weight with increasing percentages of fat is plainly shown by their tabl on p. 124 .

The difference in our results shows that while the Gerber method is accurate in the hands of those who check their results by gravimetric methods, the conditions are not sufficiently deined to make it a really accurate method, and the results by 
unskilled persons may have considerable error, and it appears that the figure (for one division $=0.125$ c.c.) adopted by the standardising authorities, which is a mean of the figures of Messrs. Day and Grimes and my own, is a perfectly satisfactory one for use in standardising Gerber butyrometers.

Messrs. Day and Grimes write:

In his criticism of our paper, Richmond is partly correct in pointing out an error in our formula, in which the density used should have been the true density at the temperature of reading. This makes our calculated graduation-volume about 0.2 per cent. too low; Richmond's alteration would make it 0.1 per cent. low.

We did not overlook the effect of decrease of viscosity and surface tension on the Iraining of pipettes (p. 124, third line below Table I.).

As regards coefficient of expansion, this is the increase of volume of the mass occupying unit volume at $0^{\circ} \mathrm{C}$. for a rise of temperature of $1^{\circ}$. Richmond would use the volume of unit mass at $15^{\circ} \mathrm{C}$. as a basis of calculation. We calculated our figures on unit volume at $30^{\circ} \mathrm{C}$, as butter-fat is solid at $0^{\circ}$, and the coefficients are different for the solid and liquid fat. Our figures for the sp. gr. of butter-fat, "calculated from Richmond's data," we obtained by plotting his values for $37 \cdot 8^{\circ}, 39 \cdot 5^{\circ}$, and $100^{\circ}$ C. ("Dairy Chemistry," 1899 , p. 39), as a straight line graph, which mothod we showed to be correct.

In discussing the composition of the Gerber fat we made a misstatement, which we now correct. From Lewkowitsch's data the free fatty acids have about the same, not a greater, as we stated, molecular volume as their glycerides. This, of course, would decrease the sp. gr. of the fat; but the iodine value shows saturation of double bonds, probably by sulphuric acid, though we did not confirm this by determining the combined sulphuric acid. This would increase the sp. gr.; however, the extreme difference of sp. gr. observed between Richmond and ourselves is only 0.7 per cent.

Neither precaution mentioned by Richmond was neglected in the gravimetric determinations.

We attribute the difference between the graduation-volumes found to differences in procedure, and think that our own method (three minutes' whirling, and reading after immersion in a water-bath at $65^{\circ} \mathrm{C}$.) is in better agreement with the usual practice than is Richmond's (ten minutes' whirling, reading as near $70^{\circ} \mathrm{C}$. as possible).

Of course, the method is not accurate in the hands of unskilled persons or for curdled and abnormal milks, but we think it capable of considerable accuracy in the case of fresh samples when carried out under standard conditions.

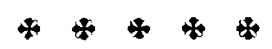

